\title{
COHERENCE INVARIANT MAPPINGS OF SYMMETRIC TRANSFORMATIONS ${ }^{1}$
}

\author{
H. G. JACOB
}

Introduction. In a previous paper [3] a characterization was found of the coherence invariant mappings of the group of linear transformations of finite rank of one vector space onto a similar group for a second vector space. In this paper we shall investigate the coherence invariant mappings of the set of symmetric (self-adjoint) transformations of finite rank on a single vector space. We assume here that $\mathfrak{X}$ is a vector space of dimension $\geqq 3$ over a field of characteristic $\neq 2$ and that $\mathfrak{X}$ is self-dual relative to a nondegenerate hermitian scalar product. The principal result obtained in this setting is that, similar to the above mentioned case, the coherence invariant mapping is essentially induced by a semilinear transformaton on $\mathfrak{X}$. L. K. Hua has proved this for the special case of symmetric matrices over a field, i.e., the case in which the scalar product is symmetric [1]. The generalization, therefore, is to the entire class of hermitian products and to infinite dimension and is made possible by an application of the fundamental theorem of projective geometry and techniques requiring commutativity of the field. The methods used differ from those employed by Hua and considerably shorten some of the arguments.

The author wishes to acknowledge his indebtedness to Professor C. E. Rickart for the many suggestions he offered during the preparation of this paper.

1. Self-dual vector spaces. Let $\mathfrak{X}$ be a left vector space of dimension $\geqq 3$ over a field $\Phi$ of characteristic $\neq 2$ and suppose that $\alpha \rightarrow \alpha^{*}$ is an involution in $\Phi$, i.e., $\alpha \rightarrow \alpha^{*}$ is an automorphism such that $\left(\alpha^{*}\right)^{*}=\alpha$. $\mathfrak{X}$ may also be regarded as a right vector space over $\Phi$ by defining $x \alpha=\alpha^{*} x$ and addition as in $\mathfrak{X}$. $\mathfrak{X}$ is said to be self-dual if there exists a nondegenerate hermitian scalar product $(x, y)$ defined on $\mathfrak{X} \times \mathfrak{X}$ to $\Phi$ having the following properties

$$
\begin{aligned}
(\alpha x+\beta y, z) & =\alpha(x, z)+\beta(y, z), \\
(x, y) & =0 \text { for all } x \text { implies } y=0, \\
(y, x) & =(x, y)^{*} .
\end{aligned}
$$

Presented to the Society, October 27, 1956; received by the editors February 9, 1957.

1 Prepared in part under a grant-in-aid from Louisiana State University. 
As a consequence of (i) and (iii) $(z, \alpha x+\beta y)=(z, x) \alpha^{*}+(z, y) \beta^{*}$. Also Jacobson [4] has shown that if $x_{1}, \cdots, x_{n}$ are linearly independent elements of $\mathfrak{X}$, then there exist elements $y_{1}, \cdots, y_{n}$ in $\mathfrak{X}$ such that $\left(x_{i}, y_{j}\right)=\delta_{i j}$ where $\delta_{i j}$ is the Kronecker delta.

If $T$ is a linear transformation on $\mathfrak{X}$ the transformation $T^{*}$ such that $(x T, y)=\left(x, y T^{*}\right)$ is called the adjoint of $T$ and is unique when it exists. A transformation $T$ is said to be of finite rank $n$ if its range $\mathfrak{X} T$ is of dimension $n$. We shall denote this by writing $r(T)=n$. It can be shown that every transformation of finite rank has the form $x T=\sum_{i=1}^{n}\left(x, x_{i}\right) y_{i}$ where the sets $x_{1}, \cdots, x_{n}$ and $y_{1}, \cdots, y_{n}$ are each linearly independent in $\mathfrak{X}[4]$, furthermore $T^{*}$ exists and has the form $x T^{*}=\sum_{i=1}^{n}\left(x, y_{i}\right) x_{i}$. Hereafter, we shall write $T$ $=\sum_{i=1}^{n} x_{i} \otimes y_{i}$ and $T^{*}=\sum_{i=1}^{n} y_{i} \otimes x_{i}$ when $r(T)=n$ and regard $T$ as an element of the Kronecker product $\mathfrak{X} \otimes \mathfrak{X}$ as described in [3]. We then have $\alpha x \otimes y=x \otimes \alpha^{*} y$. A transformation $T$ is said to be symmetric (self-adjoint) if $T=T^{*}$.

LEMma 1.1. If $T$ is a symmetric transformation of finite rank then $T=\sum_{i, j=1}^{n} \alpha_{i j} x_{j} \otimes x_{i}$ where $\alpha_{i j}^{*}=\alpha_{j i}$.

Proof. Let $T=\sum_{i=1}^{n} y_{i} \otimes x_{i}$, then since $T=T^{*}$ we also have $T=\sum_{i=1}^{n} x_{i} \otimes y_{i}$. Select $z_{j}, j=1,2, \cdots, n$ so that $\left(z_{j}, x_{i}\right)=\delta_{j i}$. Then $\sum_{i=1}^{n}\left(z_{j}, y_{i}\right) x_{i}=y_{j}$ and we may write $T=\sum_{i=1}^{n} \alpha_{i j} x_{j} \otimes x_{i}$. Now $z_{k} T$ $=\sum_{i=1}^{n} \alpha_{i k}^{*} x_{i}$ and $z_{k} T^{*}=\sum_{i=1}^{n} \alpha_{k i} x_{i}$, hence it follows that $\alpha_{i k}^{*}=\alpha_{k i}$.

We note that the matrix $A=\left(\alpha_{i j}\right)$ associated with $T$ above has rank $n$ and satisfies the condition $A^{*}=A$, where $A^{*}=\left(\beta_{i j}\right), \beta_{i j}=\alpha_{j i}^{*}$. Also we remark when $r(T)=1$ that $T=\alpha x \otimes x$, where $\alpha^{*}=\alpha$.

LEMMA 1.2. Every symmetric transformation $T$ of finite rank is of the form $T=\sum_{i=1}^{n} \alpha_{i} x_{i} \otimes x_{i}$ with $\alpha_{i}^{*}=\alpha_{i}$. $T$ is of rank $n$ if and only if $x_{1}, \cdots, x_{n}$ are linearly independent.

PROOF. Suppose that $T=\sum_{i=1}^{n} \alpha_{i j} x_{j} \otimes x_{i}=\sum_{i=1}^{n} \beta_{k l} y_{l} \otimes y_{k}$, then we shall show that $A=C^{*} B C$ where $A=\left(\alpha_{i j}\right), B=\left(\beta_{k l}\right)$, and $C=\left(\gamma_{k j}\right)$ is the matrix associated with the transformation $y_{k}=\sum_{i=1}^{n} \gamma_{k j} x_{j}$. For,

$$
\begin{aligned}
\sum_{k, l=1}^{n} \beta_{k l} y_{l} \otimes y_{k} & =\sum_{k, l=1}^{n} \beta_{k l} y_{l} \otimes \sum_{i=1}^{n} \gamma_{k i} x_{i}=\sum_{k, l, i=1}^{n}{ }_{\gamma_{k i} \beta_{k l} y_{l} \otimes x_{i}}^{*} \\
& =\sum_{k, l, i, j=1}^{n} \underset{\gamma_{k i} \beta_{k l} \gamma_{l j} x_{j} \otimes x_{i} .}{ }
\end{aligned}
$$

Thus $\alpha_{i j}=\sum_{k, l=1}^{n} \gamma_{k i}^{*} \beta_{k l} \gamma_{l j}$. Now since $B$ may be regarded as the matrix of a nondegenerate hermitian form on an $n$-dimensional space and it is well known that there exists a nonsingular matrix $C$ such that $C^{*} B C$ has diagonal form [4], the conclusion is readily seen. The 
second statement is established by using the elements $y_{1}, \cdots, y_{n}$ in $\mathfrak{X}$ such that $\left(x_{i}, y_{j}\right)=\delta_{i j}$. Then $y_{j} T=\left(y_{j}, \alpha_{j} x_{j}\right) x_{j}=\alpha_{j} x_{j}$.

2. Coherence invariant mappings. Assume hereafter that $\mathfrak{X}$ is a self-dual vector space over a field $\Phi$ and let $\subseteq$ denote the set of symmetric transformations of finite rank. A one-to-one mapping $\sigma$ of $\mathfrak{S}$ onto itself is said to be coherence invariant, if, for $T_{1}, T_{2} \in \mathfrak{S}, r\left(T_{1}^{\sigma}-T_{2}^{\sigma}\right)$ $=1$ whenever $r\left(T_{1}-T_{2}\right)=1$, and $\sigma^{-1}$ possesses the same property. It follows at once from this definition that if $\sigma$ maps 0 on 0 then the subset of elements of rank one of $\subseteq$ is mapped onto itself. In fact, if $r\left(T_{1}-T_{2}\right)=n$ then $r\left(T_{1}^{\sigma}-T_{2}^{\sigma}\right)=n$. This is a consequence of the fact that for $T=\sum_{i=1}^{n} \alpha_{i} x_{i} \otimes x_{i}, T \in \mathcal{S}$, there exists a sequence of symmetric elements $T_{j}, j=0,1, \cdots, n$, namely $T_{0}=0$ and $T_{j}$ $=\sum_{1}^{j} \alpha_{i} x_{i} \otimes x_{i}$, such that $r\left(T_{j+1}-T_{j}\right)=1$. Furthermore, no shorter sequence with this property exists. Since the most general coherence invariant mapping may be obtained from one in which 0 maps onto 0 we shall consider only this type.

Now if $S$ is a nonsingular semi-linear transformation of $\mathfrak{X}$ with an associated automorphism $\tau$ of $\Phi$ and $\lambda$ is a scalar such that $\lambda^{*}=\lambda$ and $\lambda_{e}$ denotes the linear transformation obtained by left multiplication of $\lambda$, it is readily seen that $\sum \alpha_{i} x_{i} \otimes x_{i} \rightarrow \sum\left(\alpha_{i} x_{i}\right) S \lambda_{e} \otimes x_{i} S=\sum \lambda \alpha_{i}^{\tau} x_{i} S$ $\otimes x_{i} S$ is a coherence invariant mapping of $\subseteq$ when $\tau$ commutes with the involution $\alpha \rightarrow \alpha^{*}$. It suffices to show this must be the case for an element of rank one. When the latter condition holds the mapping is well defined and will be denoted by $S \lambda_{e} \otimes S$. Our goal is to show that every coherence invariant mapping is so induced.

Consider next a maximal set of mutually coherent symmetric transformations of rank one, which we shall call a maximal set of rank one. From the definition of coherence and the remark after Lemma 1.1 it is evident that such a set is comprised of all transformations $T=\alpha x \otimes x$ for some fixed $x$ and $\alpha$ variable subject to the restriction that $\alpha^{*}=\alpha$ (nonzero self-adjoint elements of $\Phi$ ). If another representation of $T$ is used, for example $T=\beta y \otimes y$, then it is readily seen that in this case $y=\lambda x$, and so there is a one-to-one correspondence between the maximal sets of rank one and the one dimensional subspaces of $\mathfrak{X}$. We remark that two distinct maximal sets of rank one are disjoint. Now under a coherence invariant transformation $\sigma$ a maximal set of rank one must be mapped onto a similar set and distinct sets have distinct images, hence $\sigma$ induces a one-to-one mapping of the one dimensional subspaces of $\mathfrak{X}$. If $[x]$ denotes the one-dimensional space generated by $x$ and $T^{\sigma}$ is written $T^{\sigma}=\beta x^{\sigma} \otimes x^{\sigma}$, then the induced mapping will be given by $[x] \rightarrow\left[x^{\sigma}\right]$. In Lemma 2.3 we shall prove that $\left[(x+y)^{\sigma}\right] \subseteq\left[x^{\sigma}\right]+\left[y^{\sigma}\right]$. Finally it should be noted that 
if any pair of representatives $z \in[x]$ and $z^{\sigma} \in\left[x^{\sigma}\right]$ are selected and then held fixed the image of $T=\alpha z \otimes z$ may be expressed as $T^{\circ}$ $=\alpha_{z} z^{\sigma} \otimes z^{\sigma}$, where $\alpha \rightarrow \alpha_{z}$ is also one-to-one on the self-adjoint elements of $\Phi$ and dependent on the pair $z$ and $z^{\sigma}$.

Lemma 2.1. If $(\alpha x \otimes x)^{\sigma}=\alpha_{x} x^{\sigma} \otimes x^{\sigma}$ and $(\beta y \otimes y)^{\sigma}=\beta_{y} y^{\sigma} \otimes y^{\sigma}$ where $x$ and $y$ are linearly independent, then $(\alpha x \otimes x+\beta y \otimes y)^{\sigma}=\alpha_{x} x^{\sigma} \otimes x^{\sigma}+\beta_{y} y^{\sigma}$ $\otimes y^{\sigma}$.

PRoof. Let $T=\alpha x \otimes x+\beta y \otimes y$. Since $r(T)=2$ and $r(T-\alpha x \otimes x)=1$ $=r\left(T^{\sigma}-\alpha_{x} x^{\sigma} \otimes x^{\sigma}\right)$ we may write $T^{\sigma}=\alpha_{x} x^{\sigma} \otimes x^{\sigma}+\gamma z \otimes z$, where $z$ is independent of $x^{\sigma}$ but dependent on $x^{\sigma}$ and $y^{\sigma}$ because $r(T-\beta y \otimes y)$ $=1$. Suppose that $\beta$ varies over the nonzero self-adjoint elements of $\Phi$ while $\alpha, x$, and $y$ remain constant, then we claim $T^{\sigma}$ is of the form $T^{\sigma}=\alpha_{x} x^{\sigma} \otimes x^{\sigma}+\gamma^{(\beta)} z \otimes z$, where $z$ is fixed and $\gamma^{(\beta)}$ varies over the set of nonzero self-adjoint elements; in fact $\beta \rightarrow \gamma^{(\beta)}$ is one-to-one and onto for the set. For, if $T_{1}=\alpha x \otimes x+\beta_{1} y \otimes y$ and $T_{2}=\alpha x \otimes x+\beta_{2} y \otimes y$, then $r\left(T_{1}-T_{2}\right)=1$. Thus $T_{1}^{\sigma}=\alpha_{x} x^{\sigma} \otimes x^{\sigma}+\gamma_{1} z_{1} \otimes z_{1}$, also $T_{2}^{\sigma}=\alpha_{x} x^{\sigma} \otimes x^{\sigma}+\gamma_{2} z_{2}$ $\otimes z_{2}$, and $r\left(T_{1}^{\sigma}-T_{2}^{\sigma}\right)=1$ which imply $z_{1}=\lambda z_{2}$; if not $r\left(T_{1}^{\sigma}-T_{2}^{\sigma}\right)=2$. Assume, therefore, that $\alpha, x, y$, and $z$ are fixed and $z=\delta x^{\sigma}+\eta y^{\sigma}$ where $\eta \neq 0$, otherwise $r\left(T^{\sigma}\right)=1$. As a result of these assumptions we have,

$$
\begin{aligned}
T^{\sigma}-\beta_{y} y^{\sigma} \otimes y^{\sigma}= & \gamma^{(\beta)}\left(\delta x^{\sigma}+\eta y^{\sigma}\right) \otimes\left(\delta x^{\sigma}+\eta y^{\sigma}\right)+\alpha_{x} x^{\sigma} \otimes x^{\sigma}-\beta_{y} y^{\sigma} \otimes y^{\sigma} \\
= & {\left[\delta^{*} \gamma^{(\beta)} \delta x^{\sigma}+\delta^{*} \gamma^{(\beta)} \eta y^{\sigma}+\alpha_{x} x^{\sigma}\right] \otimes x^{\sigma} } \\
& +\left[\eta^{*} \gamma^{(\beta)} \delta x^{\sigma}+\eta^{*} \gamma^{(\beta)} \eta y^{\sigma}-\beta_{y} y^{\sigma}\right] \otimes y^{\sigma}
\end{aligned}
$$

where $\gamma^{(\beta)}$ varies with $\beta$. Assume next that $\delta \neq 0$. For $r\left[T^{\sigma}-\beta_{y} y^{\sigma} \otimes y^{\sigma}\right]$ to equal 1 the following relation must hold

$$
\left(\delta^{*} \gamma^{(\beta)} \delta+\alpha_{x}\right) x^{\sigma}+\delta^{*} \gamma^{(\beta)} \eta y^{\sigma}=\lambda^{(\beta)}\left[\eta^{*} \gamma^{(\beta)} \delta x^{\sigma}+\left(\eta^{*} \gamma^{(\beta)} \eta-\beta_{y}\right) y^{\sigma}\right]
$$

for all $\beta \neq 0$.

For some $\beta=\beta_{0}, \delta^{*} \gamma^{\left(\beta_{0}\right)} \delta=-\alpha_{x}$ which implies $\lambda^{\left(\beta_{0}\right)} \eta^{*} \gamma^{\left(\beta_{0}\right)} \delta x^{\sigma}=0$. Thus $\lambda^{\left(\beta_{0}\right)}=0$ and $\delta^{*} \gamma^{\left(\beta_{0}\right)} \eta=0$ which is a contradiction since neither $\eta$ nor $\gamma^{\left(\beta_{0}\right)}$ is zero. We must have $\delta=0$ and $T^{\sigma}=\alpha_{x} x^{\sigma} \otimes x^{\sigma}+\beta_{y} y^{\sigma} \otimes y^{\sigma}$.

LEMMA 2.2. If $T=\sum_{i=1}^{n} \alpha_{i} x_{i} \otimes x_{i}$ is symmetric of rank $n$, then $T^{\sigma}=\sum_{i=1}^{n} \alpha_{x_{i}} x_{i}^{\sigma} \otimes x_{i}^{\sigma}$, where $\left(\alpha_{i} x_{i} \otimes x_{i}\right)^{\sigma}=\alpha_{x_{i}} x_{i}^{\sigma} \otimes x_{i}^{\sigma}$.

Proof. The lemma is true for $n=1,2$ by the above result and we are able to prove it for all $n$ by induction. Assume the conclusion for $n-1$. Since $r\left(T-\sum_{2}^{n} \alpha_{i} x_{i} \otimes x_{i}\right)=1$ we may write $T^{\sigma}$ $=\beta y \otimes y+\sum_{2}^{n} \alpha_{x_{i}} x_{i}^{\sigma} \otimes x_{i}^{\sigma}$. Also $r\left(T-\sum_{1}^{n-1} \alpha_{i} x_{i} \otimes x_{i}\right)=1$ from which we see that $r\left(T^{\sigma}-\sum_{1}^{n-1} \alpha_{x_{i}} x_{i}^{\sigma} \otimes x_{i}^{\sigma}\right)=r\left(\beta y \otimes y+\alpha_{x_{n}} x_{n}^{\sigma} \otimes x_{n}^{\sigma}-\alpha_{x_{1}} x_{1}^{\sigma} \otimes x_{1}^{\sigma}\right)$ $=1$. Therefore $y=\lambda_{1} x_{1}^{\sigma}+\lambda_{n} x_{n}^{\sigma}$. Similarly we may show that $y=\delta_{1} x_{1}^{\sigma}$ $+\delta_{n-1} x_{n-1}^{\sigma}$. Obviously $\lambda_{n}=0$ and $T^{\sigma}=\sum_{i=1} \alpha_{x_{i}} x_{i}^{\sigma} \otimes x_{i}^{\sigma}$. 
Note that this argument does not hold for $n=2$.

Lemma 2.3. If $x, y \in \mathfrak{X}$ are linearly independent then $\left[(x+y)^{\sigma}\right]$ $\subseteq\left[x^{\sigma}\right]+\left[y^{\sigma}\right]$.

Proof. Let $T=x+y \otimes x+y=x \otimes x+y \otimes y+x \otimes y+y \otimes x$, thus $r(T-x \otimes x-y \otimes y)=2$ which implies $r\left(T^{\sigma}-\alpha x^{\sigma} \otimes x^{\sigma}-\beta y^{\sigma} \otimes y^{\sigma}\right)=2$ where $(x \otimes x)^{\sigma}=\alpha x^{\sigma} \otimes x^{\sigma}$ and $(y \otimes y)^{\sigma}=\beta y^{\sigma} \otimes y^{\sigma}$. Now if $T^{\sigma}=\gamma(x+y)^{\sigma}$ $\otimes(x+y)^{\sigma}$ we must conclude that $(x+y)^{\sigma}$ is linearly dependent on $x^{\sigma}$ and $y^{\sigma}$.

The last lemma asserts that the induced mapping (by $\sigma$ ) of the one dimensional subspaces satisfies the hypotheses of the fundamental theorem of projective geometry (for the particular form used here see Rickart [6]). We may therefore say that the mapping $[x] \rightarrow\left[x^{\sigma}\right]$ is induced by a nonsingular semi-linear transformation $S$ on $\mathfrak{X}$. Employing this fact we may express the image of $T=\alpha x \otimes x$ as $T^{\sigma}=\alpha_{x} x S \otimes x S$, where $\alpha_{x}$ is now dependent on $x$ only. At this point we are not permitted to assume that $T^{\sigma}=(\alpha x) S \otimes x S=\alpha^{\tau} x S \otimes x S$, or equivalently that $\alpha_{x}=\alpha^{\tau}$, where $\tau$ is the automorphism associated with $S$. This is true even if it were known that $\tau$ commutes with $\alpha \rightarrow \alpha^{*}$, but, of course the latter has not yet been established.

Lemma 2.4. If $x, y \in \mathfrak{X}$ are linearly independent then $\alpha x \otimes x+\beta y \otimes y$ $=\gamma(x+y) \otimes(x+y)+\delta(x-y) \otimes(x-y)$ implies $\alpha=\beta=2 \gamma=2 \delta$.

Proof. Rewriting the equality we obtain

$$
[\alpha x-\gamma x-\gamma y-\delta x+\delta y] \otimes x=[\gamma x+\gamma y-\delta x+\delta y-\beta y] \otimes y
$$

which implies $\gamma=\delta$ and $\alpha=2 \gamma$. The last implications follow from the fact that if $z \otimes x=w \otimes y$ for $x$ and $y$ independent then $z=0=w$, otherwise there exists $u \in \mathfrak{X}$ such that $(u, z) \neq 0$ which leads to a contradiction.

By means of Lemma 2.4 we may show that the mapping $\alpha \rightarrow \alpha_{x}$ for $\alpha^{*}=\alpha$ is actually independent of $x$ and also that $\alpha / 2 \rightarrow \alpha_{x} / 2$. This is seen by again considering $x$ and $y$ independent in $\mathfrak{X}$. If

$$
T=\alpha x \otimes x+\alpha y \otimes y=\frac{\alpha}{2}(x+y) \otimes(x+y)+\frac{\alpha}{2}(x-y) \otimes(x-y),
$$

then from what has previously been done, we have

$$
\begin{aligned}
T^{\sigma}= & \alpha_{x} x S \otimes x S+\alpha_{y} y S \otimes y S=(\alpha / 2)_{x+y}(x S+y S) \otimes(x S+y S) \\
& +(\alpha / 2)_{x-y}(x S-y S) \otimes(x S-y S),
\end{aligned}
$$

whence $\alpha_{x}=\alpha_{y}=2(\alpha / 2)_{x+y}=2(\alpha / 2)_{x-y}$. Since the dimension of $\mathfrak{X} \geqq 3$ 
the desired conclusion is obtained. To make the remaining arguments somewhat easier to read we shall follow the mapping $\sigma$ by the mapping $\left.\xi=\left(1^{\prime}\right)_{e}^{-1}\right) \otimes I$, where $1^{\prime}$ is the image of $1 \in \Phi$ and $I$ is the identity mapping. Thus $(x \otimes x)^{\sigma \xi}=x S \otimes x S$ for all $x \in \mathfrak{X}$, and we may write $(\alpha x \otimes x)^{\sigma \xi}=\alpha^{\prime} x S \otimes x S$, where $\alpha \rightarrow \alpha^{\prime}$ denotes the induced mapping of the self adjoint elements of $\Phi$. It is apparent since $\alpha x \otimes \alpha x=\alpha^{*} \alpha x \otimes x$ that $(\alpha x \otimes \alpha x)^{\sigma \xi}=(\alpha x) S \otimes(\alpha x) S=\alpha^{\tau *} \alpha^{\tau} x S \otimes x S$. Also $\left(\alpha^{*} \alpha x \otimes x\right)^{\sigma \xi}$ $=\left(\alpha^{*} \alpha\right)^{\prime} x S \otimes x S$, hence $\alpha^{\tau *} \alpha^{\tau}=\left(\alpha^{*} \alpha\right)^{\prime}$ for all $\alpha$, and, in particular, $\alpha^{\tau *} \alpha^{\tau}=\left(\alpha^{2}\right)^{\prime}$ when $\alpha^{*}=\alpha$. We next prove the following.

LEMMA 2.5. The mapping $\alpha \rightarrow \alpha^{\prime}$ is additive.

Proof. Suppose $x$ and $y$ are linearly independent and that

$$
\begin{aligned}
T= & \left(\alpha_{1}+\alpha_{2}\right) x \otimes x+\alpha_{1} y \otimes y=\frac{\alpha_{1}}{2}(x+y) \otimes(x+y) \\
& +\frac{\alpha_{1}}{2}(x-y) \otimes(x-y)+\alpha_{2} x \otimes x .
\end{aligned}
$$

Thus

$$
T^{\sigma \xi}=\beta x S \otimes x S+\alpha_{2}^{\prime} x S \otimes x S+\alpha_{1}^{\prime} y S \otimes y S,
$$

or

$$
T^{\sigma \xi}=\gamma z \otimes z+\frac{\alpha_{1}^{\prime}}{2}(x S-y S) \otimes(x S-y S)+\alpha_{2}^{\prime} x S \otimes x S,
$$

since $r\left(T-\alpha_{1}(x-y) / 2 \otimes(x-y)-\alpha_{2} x \otimes x\right)=1$. The rank of $T$ is 2 which means that $z$ is a linear combination of $x S$ and $y S$. Therefore, if $z=\lambda_{1} x S+\lambda_{2} y S$, we obtain by equating the two forms of $T^{\sigma \xi}$ that

$$
\begin{aligned}
\beta x S \otimes x S+\alpha_{1}^{\prime} y S \otimes y S= & {\left[\lambda_{1}^{*} \gamma\left(\lambda_{1} x S+\lambda_{2} y S\right)+\frac{\alpha_{1}^{\prime}}{2}(x S-y S)\right] \otimes x S } \\
& +\left[\lambda_{2}^{*} \gamma\left(\lambda_{1} x S+\lambda_{2} y S\right)-\frac{\alpha_{1}^{\prime}}{2}(x S-y S)\right] \otimes y S .
\end{aligned}
$$

From this equation one has

$$
\begin{aligned}
\beta x S & =\left(\lambda_{1}^{*} \gamma \lambda_{1}+\frac{\alpha_{1}^{\prime}}{2}\right) x S+\left(\lambda_{1}^{*} \gamma \lambda_{2}-\frac{\alpha_{1}^{\prime}}{2}\right) y S, \\
\alpha_{1}^{\prime} y S & =\left(\lambda_{2}^{*} \gamma \lambda_{1}-\frac{\alpha_{1}^{\prime}}{2}\right) x S+\left(\lambda_{2}^{*} \gamma \lambda_{2}+\frac{\alpha_{1}^{\prime}}{2}\right) y S .
\end{aligned}
$$

Hence, $\lambda_{1}^{*} \gamma \lambda_{2}-\alpha_{1}^{\prime} / 2=0, \quad \lambda_{1}^{*} \gamma \lambda_{1}+\alpha_{1}^{\prime} / 2=\beta$, and $\lambda_{2}^{*} \gamma \lambda_{2}+\alpha_{1}^{\prime} / 2=\alpha_{1}^{\prime}$. These combine to show $\alpha_{1}^{\prime} / 2=\lambda_{2}{ }^{*} \gamma \lambda_{2}=\lambda_{1}{ }^{*} \gamma \lambda_{2}$ which in turn implies $\lambda_{1}=\lambda_{2}$ and $\beta=\alpha_{1}^{\prime}$. The mapping $\alpha \rightarrow \alpha^{\prime}$ is therefore additive. 
LEMMA 2.6. $\alpha^{\prime}=\alpha^{\tau}$ for all $\alpha=\alpha^{*}$ in $\Phi$ and $\gamma^{\tau *}=\gamma^{*_{\tau}}$ for all $\gamma \in \Phi$.

Proof. Assume that $\alpha$ and $\beta$ are self adjoint elements of $\Phi$. Since $\alpha+\beta$ is also self adjoint $(\alpha+\beta)^{\tau *}(\alpha+\beta)^{\tau}=\left[(\alpha+\beta)^{2}\right]^{\prime}, \alpha^{\tau *} \alpha^{\tau}+\beta^{\tau *} \alpha^{\tau}$ $+\alpha^{\tau *} \beta^{r}+\beta^{\tau *} \beta^{r}=\left(\alpha^{2}\right)^{\prime}+2(\alpha \beta)^{\prime}+\left(\beta^{2}\right)^{\prime}$ since $\Phi$ is a field. From previously determined identities it is immediate that $\beta^{r *} \alpha^{r}+\alpha^{\tau *} \beta^{r}=2(\alpha \beta)^{\prime}$. Let $\beta=\alpha^{-1}$ then $\alpha^{\tau} / \alpha^{\tau *}+\alpha^{\tau *} / \alpha^{\tau}=2$, consequently $\left(\alpha^{\tau}-\alpha^{\tau *}\right)^{2}=0$ hence $\alpha^{\tau}=\alpha^{\tau *}$ for all $\alpha=\alpha^{*}$, and $2 \alpha^{\tau} \beta^{\tau}=2(\alpha \beta)^{\prime}$. Next let $\beta=1$ to obtain $\alpha^{\tau}=\alpha^{\prime}$. Finally $\left(\gamma^{*} \gamma\right)^{\prime}=\gamma^{*} \gamma^{\tau}$ for all $\gamma \in \Phi$, as was previously stated, which yields $\left(\gamma^{*} \gamma\right)^{\prime}=\left(\gamma^{*} \gamma\right)^{r}=\gamma^{* \tau} \gamma^{\tau}$ and $\gamma^{* *}=\gamma^{* \tau}$.

Having observed that $\tau$ commutes with $\alpha \rightarrow \alpha^{*}$ we may now speak of the mapping $S \otimes S$ which is well defined and state the principal theorem.

THEOREM 2.1. Let $\mathfrak{X}$ be a vector space of dimension $\geqq 3$ over a field $\Phi$ of characteristic $\neq 2$. Let $\mathfrak{X}$ be self-dual relative to a nondegenerate hermitian scalar product with associated involution $\alpha \rightarrow \alpha^{*}$ and $\subseteq$ be the set of symmetric transformations on $\mathfrak{X}$ of finite rank. If $\sigma$ is a coherence invariant mapping of $\subseteq$ onto itself there exists a nonsingular semi-linear transformation $S$ on $\mathfrak{X}$ with associated automorphism $\tau$ and a self-adjoint element $\lambda=\lambda^{*} \in \Phi$ such that $T^{\sigma}=T\left[S \lambda_{e} \otimes S\right]$ for all $T \in \subseteq$ S. Moreover, $\gamma^{* r}=\gamma^{*}$ for $\gamma \in \Phi$.

REMARK. Theorem 2.1 can be applied to obtain another proof of a theorem concerning the extensibility of Jordan automorphisms on the Jordan ring of symmetric elements of an involutorial primitive ring with minimal ideals [5]. A simple modification of Hua's characterization of the elements of rank one [2] to take care of symmetric elements, coupled with the fact that such a primitive ring is locally canonical-matrix permits one to extend the Jordan automorphism uniquely to the entire ring under the assumption that the ring arises from an hermitian product.

\section{BIBLIOGRAPHY}

1. L. K. Hua, Geometry of symmetric matrices over a field of characteristic other than two, Ann. of Math. vol. 50 (1949) pp. 8-31.

2. - A theorem on matrices over a field and its applications, Journal of the Chinese Mathematics Society vol. 1 (1951) pp. 110-163.

3. H. G. Jacob, Coherence invariant mappings on Kronecker products, Amer. J. Math. vol. 77 (1955) pp. 177-189.

4. N. Jacobson, Lectures in abstract algebra, vol. II, New York, 1953.

5. N. Jacobson and C. E. Rickart, Homomorphisms of Jordan rings of self-adjoint elements, Trans. Amer. Math. Soc. vol. 72 (1952) pp. 310-322.

6. C. E. Rickart, Isomorphic groups of linear transformations, Amer. J. Math. vol. 72 (1950) pp. 451-464. 